\title{
Improved SURF Algorithm Based On ACO
}

\author{
Chao Chen ${ }^{1, \text { a }}$, Xiaohong Wang ${ }^{1}$, Shizheng Zhou ${ }^{1}$ \\ ${ }^{1}$ Department of Computer Science and Technology, Communication University of China, Beijing \\ 100024, China \\ achen_chao@cuc.edu.cn
}

Keywords: image registration; interest points; SURF; image edge detection; ACO

\begin{abstract}
SURF (Speeded-Up Robust Features) is a scale- and rotation-invariant algorithm, which has a better repeatability, distinctiveness, robustness, and a faster computing and comparing speed. In this paper, we propose an improved SURF algorithm based on ACO (Ant Colony Optimization). First of all, the algorithm uses SURF to find all interest points. Secondly, each pixel of the original image is seen as an ant, imitates the process of ants search food to get the image edge. Finally, selects the interest points from the area around the image edge. The experimental results show that the interest points extracted by the improved SURF algorithm based on ACO are more robust, and the number of them is effectively reduced. In this way, we can reduce the amount of calculation for the subsequent image registration.
\end{abstract}

\section{Introduction}

Image registration is a hot issue in the field of remote sensing data analysis, computer vision, and image processing, and so on. It is a process of two or more images matching or overlaying, the images are obtained in different time, different sensors (imaging equipment) or different conditions (weather, illumination, camera position and angle, etc.). At present, it is widely used, including 3D reconstruction, image retrieval, object recognition, and so on.

Generally, image registration can be classified into image registration based on gray scale and image registration based on features. Image registration based on gray scale usually directly uses gray information of the whole image, and thinks that corresponding points and surrounding areas of corresponding points between images are with the same or similar gray value, so it makes gray similarity the basis of the definition of similarity measure, then designs corresponding search strategy to find the optimal image geometry transform parameter which can make similarity measure function reach a maximum. For the reason it needs to calculate the gray value of the points in the area around the matching points, its computation amount is large, and also has a slower speed. While image registration based on features extracts features remain unchanged in the image, such as edge point, the center of closed region, and so on, as the reference information of image registration. Using this kind of method, the main advantage is that it extracts remarkable features of the image, greatly compresses the image information, and has good robustness, small amount of calculation, fast speed. Image registration based on features has already become the mainstream of the image registration research direction [1].

SURF (Speeded-Up Robust Features) [2], proposed in 2006 by Herbert Bay, is one of the feature-based image registration method, including two parts, interest point detection and interest point description. This algorithm is proposed on the basis of SIFT (Scale-Invariant Feature Transform) algorithm [3]. The same as SIFT, the features extracted by SURF also have scale- and rotation-invariant performance, and with respect to illumination change, affine and perspective transformation, it has partial invariance. In repeatability, distinctiveness, and robustness these three aspects, SURF all exceeds or approaches the previously proposed such kind of methods, and there is obvious advantage in the speed of computing [4].

In this paper, we use SURF to find interest points, and then use ACO (Ant Colony Optimization) [5] to detect image edges [6], select the interest points around the area of the image edge as the final interest points. The next section introduces interest point extracting based on SURF. The third section 
introduces interest point selecting based on ACO. The forth section verifies the validity of the algorithm through experiments. The article is concluded in the fifth section.

\section{Interest point extracting based on SURF}

The SURF-based interest point extracting method consists of two parts, including interest point detection and interest point description and matching.

Interest Point Detection. Interest point detection generally involves three steps, the establishment of integral image, the establishment of image's scale space based on box filters, and interest point location.

Each pixel of integral images represents the sum of all pixels of the original image in a rectangular area from the origin to the pixel. The introduction of integral images solves the rapid calculation of a rectangular area, and the approximation of box filters greatly improves the computing speed. Box filters are used to approximate Gaussian kernel function, which make convolution templates only consist of simple rectangular regions.

Hessian matrix is defined as

$$
H(x, \sigma)=\left[\begin{array}{ll}
L_{x x}(x, \sigma) & L_{x y}(x, \sigma) \\
L_{x y}(x, \sigma) & L_{y y}(x, \sigma)
\end{array}\right],
$$

where $\mathrm{x}=(\mathrm{x}, \mathrm{y})$ is a point of image $\mathrm{I}, \sigma$ is scale, $\mathrm{L}_{\mathrm{xx}}(\mathrm{x}, \sigma)$ is the convolution of the Gaussian second order derivative $\partial^{2} \mathrm{~g}(\sigma) / \partial \mathrm{x}^{2}$ with $\mathrm{I}$ in $\mathrm{x}$, and similarly for $\mathrm{L}_{\mathrm{xy}}(\mathrm{x}, \sigma)$ and $\mathrm{L}_{\mathrm{yy}}(\mathrm{x}, \sigma)$.

To reduce computation, here we make an approximation, we denote $\mathrm{D}_{\mathrm{xx}}$ as the convolution of box filters with I, and similarly for $\mathrm{D}_{\mathrm{xy}}$ and $\mathrm{D}_{\mathrm{yy}}$. The $9 \times 9$ box filters are approximations of a Gaussian with $\sigma=1.2$.

The determinant of Hessian matrix can be approximated as

$$
\operatorname{det}\left(H_{\text {approx }}\right)=D_{x x} D_{y y}-\left(\omega D_{x y}\right)^{2} \text {, }
$$

where $\omega$ is the weight, and $\omega \approx 0.9$.

In order to ensure image registration with the scale-invariant performance, we need to implement an image pyramid to establish the scale space, and then find interest points in different scales of the image. The establishment of scale space is to keep the size of the original image unchanged, and change the size of box filters, then use the integral image calculated from the original image to filter, thus form the image scale space.

With respect to changes of the box filter size, the scale space is divided into several octaves, and generally, each octave is subdivided into four scale layers to achieve scale-invariant, depending on the image size. The filter sizes for the first octave are $9 \times 9,15 \times 15,21 \times 21,27 \times 27$, and a second octave is computed with the filter size $15 \times 15,27 \times 27,39 \times 39,51 \times 51$, and so on. When the image is large, the step of filter size should be adjusted accordingly.

For we have already got Hessian matrix, then a threshold should be set. Only when the determinant of Hessian matrix is larger than this threshold, the next step is determined. With respect to the point which is determined in the next step, we apply a non-maximum suppression in a $3 \times 3 \times 3$ neighborhood. If the value of a point is larger than 26 other points, it will be localized as an interest point.

Interest Point Description and Matching. Interest Point Description consists of two steps, getting the main orientation to be invariant to image rotation and extraction of the descriptor.

Orientation is calculated in a circular area of radius $6 \mathrm{~s}$ centered at the interest point, where $\mathrm{s}$ is the scale. In this area, Haar wavelet responses in $\mathrm{x}$ and $\mathrm{y}$ direction is calculated and weighted with a Gaussian. By computing the sum of all responses in a sliding orientation window of size $\pi / 3$, and traversing the entire circle every 5 degrees, then computing in the same way we get 72 new orientations. We choose the longest orientation as the main orientation.

For the extraction of the descriptor, we set the interest point as the center, and construct a $20 \mathrm{~s} \times 20 \mathrm{~s}$ square region around it, at last rotate the region orientation to the main orientation. In order to make 
better use of the image spatial information, the $20 \mathrm{~s} \times 20$ s region is divided into 16 sub-regions of $4 \mathrm{~s} \times$ $4 \mathrm{~s}$, each of which we compute Haar wavelet responses and we get a four-dimensional descriptor vector

$$
\mathrm{v}=\left(\sum \mathrm{d}_{\mathrm{x}}, \sum \mathrm{d}_{\mathrm{y}}, \sum\left|\mathrm{d}_{\mathrm{x}}\right|, \sum\left|\mathrm{d}_{\mathrm{y}}\right|\right) \text {, }
$$

where $d_{x}$ is the Haar wavelet response in horizontal direction and $d_{y}$ in vertical direction. In this way, we compute all 16 sub-regions and form a 64-dimensional descriptor vector. We normalize the descriptor to make it illumination- and scale-invariant.

After the descriptor generated, we can use the trace of the Hessian matrix for fast indexing, which accelerates the matching speed.

\section{Interest point selecting based on ACO}

Ant colony algorithm, proposed by Marco Dorigo in his doctoral thesis in 1991, is a probability-based method used to find the optimal path in the graph. It is inspired from the behavior of ants find the path in the process of searching food. Image edge detection based on ACO utilizes many ants to move on a 2D image to construct a pheromone matrix. Totally it needs four processes, including initialization process, construction process, update process and decision process.

In the initialization process, a total of $K$ ants are randomly put on the image $I$, which size is $\mathrm{M}_{1} \times \mathrm{M}_{2}$. Each pixel can be seen as a node. The initial value of each component of the pheromone matrix $\tau^{(0)}$ is set to $\tau_{\text {init. }}$

In the construction process, at the $\mathrm{n}$-th construction step, select an ant randomly from $\mathrm{K}$ ants. This ant moves L steps continually from node $(1, \mathrm{~m})$ to its neighbor node $(\mathrm{i}, \mathrm{j})$ according to the transition probability

$$
\mathrm{p}_{(\mathrm{l}, \mathrm{m}),(\mathrm{i}, \mathrm{j})}^{(\mathrm{n})}=\frac{\left(\tau_{\mathrm{i}, \mathrm{j}}^{(\mathrm{n}-1)}\right)^{\alpha}\left(\eta_{\mathrm{i}, \mathrm{j}}\right)^{\beta}}{\sum_{(\mathrm{i}, \mathrm{j}) \in \Omega_{(, \mathrm{l})}}\left(\tau_{\mathrm{i}, \mathrm{j}}^{(\mathrm{n}-1)}\right)^{\alpha}\left(\eta_{\mathrm{i}, \mathrm{j}}\right)^{\beta}},
$$

where $\tau_{i, j}^{(n-1)}$ is the pheromone value of node $(i, j), \Omega_{(1, m)}$ is the neighbor node of node $(1, m), \eta_{i, j}$ is the heuristic information of node $(i, j), \alpha$ is the influence factor of the pheromone matrix, $\beta$ is the influence factor of the heuristic matrix.

In order to determine $\eta_{\mathrm{i}, \mathrm{j}}$, the following four functions are considered,

$$
\begin{aligned}
& f(x)=\lambda x, \\
& f(x)=\lambda x^{2}, \\
& f(x)=\sin \left(\frac{\pi x}{2 \lambda}\right), \\
& f(x)=\frac{\pi x \sin \left(\frac{\pi x}{\lambda}\right)}{\lambda} .
\end{aligned}
$$

In Eq. 5 and Eq. 6, $\mathrm{x} \geq 0$. In Eq. 7 and Eq. 8, $0 \leq \mathrm{x} \leq \lambda$, and in other conditions $\mathrm{f}(\mathrm{x})=0$.

In the update process, after the movement of each ant within each construction step, if node $(i, j)$ is visited, we update the pheromone matrix according to

$$
\tau_{i, j}^{(n-1)}=(1-\rho) \cdot \tau_{i, j}^{(n-1)}+\rho \cdot \Delta_{i, j}^{(k)},
$$

where $\rho$ is the evaporation rate, $\Delta^{(\mathrm{k})}{ }_{\mathrm{i}, \mathrm{j}}=\eta_{\mathrm{i}, \mathrm{j}}$. Otherwise, we remain its value. In addition, after the movement of all ants within each construction step, we update the pheromone matrix as

$$
\tau^{(n)}=(1-\psi) \cdot \tau^{(n-1)}+\psi \cdot \tau^{(0)},
$$

where $\psi$ is the pheromone decay coefficient.

In the decision process, the initial threshold $\mathrm{T}^{(0)}$ is set to the mean value of the pheromone matrix, and according to the threshold, the matrix is divided into two categories, lower and higher than $\mathrm{T}^{(0)}$. Then, the new threshold is computed as the average of the mean of each of the above two categories. Repeat this process until the threshold does not change. 
Once we get image edges, select interest points in the surrounding area around them, and for the reason they have relatively larger gradient, these points are more robust, and can inhibit the mismatch [7].

In this paper, we traverse all the pixels found by ACO at the image edge. With respect to each pixel, compute the Euclidean distance between all interest points detected by SURF previously and it. If the distance is less than or equal to one, select the interest point as the final interest point.

\section{Experimental results and analysis}

In order to verify the validity of our algorithm, we choose a grayscale image to do experiments. The platform of the experiments is MATLAB 2010a, and code with matlab language, and use the library of OpenSURF. The CPU of the computer is Core 2 Duo $2.8 \mathrm{G}$, and the memory is $4.0 \mathrm{G}$.

The grayscale image is a campus landscape taken in Communication University of China, whose size is 128 pixels by 128 pixels. SURF parameters are set as follows, Hessian response threshold is 0.0002 , number of octaves to analyze is 5 , and initial sampling step in the image is 2 . ACO parameters are set as follows, the influence of the pheromone matrix $\alpha$ is 1 , the influence of the heuristic matrix $\beta$ is 0.1 , the evaporation rate $\rho$ is 0.1 , the pheromone decay coefficient $\psi$ is 0.05 , and the parameter $\lambda$ in $\mathrm{f}(\mathrm{x})$ is 10 . Then the following images are the results.

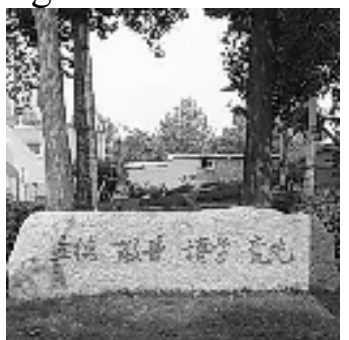

(a)

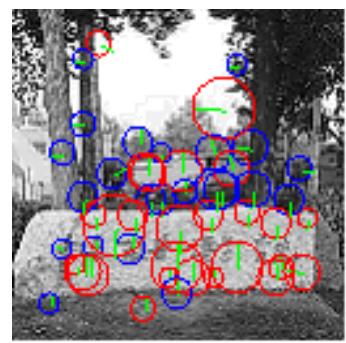

(b)

Figure 1. Image processed by SURF: (a) the original image; (b) interest points extracted from the original image.

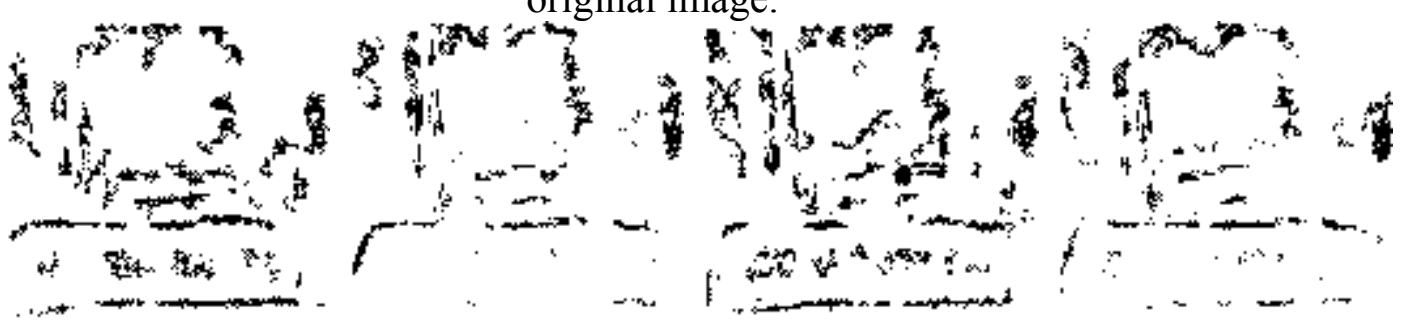

(a)

(b)

(c)

(d)

Figure 2. Image processed by ACO: (a) image edge detected with the function defined in Eq. 5; (b) image edge detected with the function defined in Eq. 6; (c) image edge detected with the function

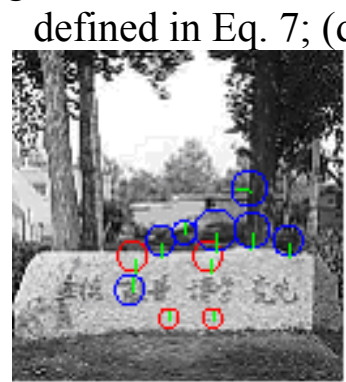

(a)

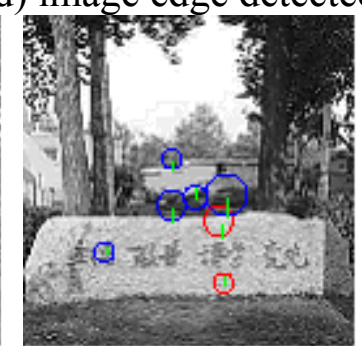

(b)

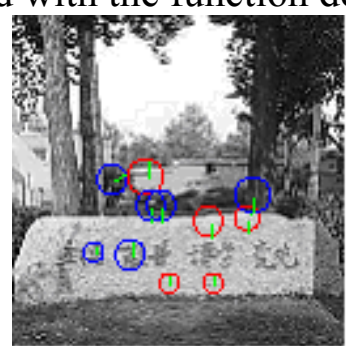

(c)

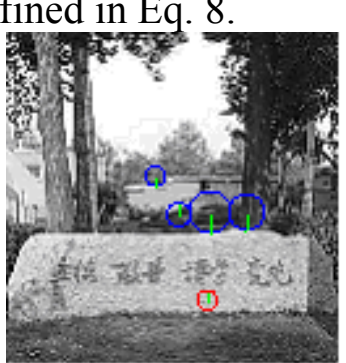

(d)

Figure 3. Image processed by our algorithm: (a) interest points selected with the function defined in Eq. 5; (b) interest points selected with the function defined in Eq. 6; (c) interest points selected with the function defined in Eq. 7; (d) interest points selected with the function defined in Eq. 8.

Table 1. Performance comparison of all algorithm

\begin{tabular}{|l|l|l|l|l|l|}
\hline Algorithm & SURF & ACO-based & ACO-based & ACO-based & ACO-based \\
\hline
\end{tabular}




\begin{tabular}{|l|l|l|l|l|l|}
\hline & & SURF in Eq. 5 & SURF in Eq. 6 & SURF in Eq. 7 & SURF in Eq. 8 \\
\hline $\begin{array}{l}\text { Number of } \\
\text { interest points }\end{array}$ & 49 & 11 & 7 & 11 & 5 \\
\hline
\end{tabular}

By comparing between Fig. 1 and Fig. 3, we can obviously see that the number of interest points is effectively reduced, and it is also shown the advantage of improved SURF algorithm based on ACO in the table above. In Fig. 2 and Fig. 3, we can also find that, the more image edge details there are, the more interest points we can select.

\section{Conclusion and outlook}

In this paper, we proposed a new SURF algorithm based on ACO, which further improves the robustness of the interest points extracted by SURF. Firstly, use SURF to detect interest points, and then get the image edge by using ACO, finally select the interest points in the edge region of the image. The experiment shows that the algorithm can effectively reduce the number of the interest points, and has a better robustness, at the same time reduces the computing time of the subsequent image registration and reduces mismatching. Although to some extent at the expense of the whole computing time, considering the algorithm is a promising simulated evolutionary and computational intelligence method, it still has some significant progress.

\section{References}

[1] Xiaoli Li, Xiaohong Wang and Chunsheng Li: Image Matching Based on Unification, The Fourth International Joint Conference on Computational Science and Optimization (2011), p. 825-828.

[2] Herbert Bay, Andreas Ess, Tinne Tuytelaars and Luc Van Gool: Speeded-Up Robust Features (SURF), Computer Vision and Image Understanding (2008), 110(3), p. 346-359.

[3] David G. Lowe: Distinctive Image Features from Scale-Invariant Keypoints, International Journal of Computer Vision (2004), 60(2), p. 91-110.

[4] Luo Juan and Oubong Gwun: A Comparison of SIFT, PCA-SIFT and SURF, International Journal of Image Processing (2009), 3(4), p. 143-152.

[5] Dorigo M., Maniezzo V. and Colorni A.: Ant System: Optimization by A Colony of Cooperating Agents, IEEE Transaction on Systems, Man, and Cybernetics-Part B (1996), 26(1), p. 29-41.

[6] Jing Tian, Weiyu Yu and Shengli Xie: An Ant Colony Optimization Algorithm for Image Edge Detection, IEEE Congress on Evolutionary Computation (2008), p. 751-756.

[7] Qiuju Yang and Xuemei Xiao: Improved SIFT Algorithm Based on Canny Feature Points, Computer Engineering and Design (2011), 32(7), p. 2428-2431, 2458. 